\title{
Transcriptional profiling of uterine leiomyoma rats treated by a traditional herb pair, Curcumae rhizoma and Sparganii rhizoma
}

\author{
Cheng Hao Yu (i) ${ }^{1,2 *}$, Jin Shuang Zhao (i) ${ }^{1,3 *}$, Hui Zhao (i) ${ }^{1 *}$, Teng Peng (i) ${ }^{1}$, Dong Cheng Shen (ii) ${ }^{4}$, \\ Quu Xia Xu(ii) ${ }^{5}$, Yao Li(ii) ${ }^{1}$, R. Clinton Webb (ii) ${ }^{2}$, Mong Heng Wang (ii) ${ }^{2}$, Xing Ming Shi (ii) ${ }^{2}$, \\ Cheng Peng (ii) ${ }^{1}$, and Wei Jun Ding (iD) \\ ${ }^{1}$ Chengdu University of Traditional Chinese Medicine, Chengdu, Sichuan, China \\ ${ }^{2}$ Medical College of Georgia, Augusta University, Augusta, GA, USA \\ ${ }^{3}$ The First Affiliated Hospital of Henan University of Traditional Chinese Medicine, Zhengzhou, Henan, China \\ ${ }^{4}$ The Community Health Service Center of Xi'an Road, Chengdu, Sichuan, China \\ ${ }^{5}$ University of Chinese Academy of Sciences Shenzhen Hospital, Shenzhen, Guangdong, China
}

\begin{abstract}
The aim of this study was to elucidate the concise effects of a traditional herb pair, Curcumae rhizoma-Sparganii rhizoma (CRSR), on uterine leiomyoma (UL) by analyzing transcriptional profiling. The UL rat model was made by intramuscular injection of progesterone and gavage administration of diethylstilbestrol. From 11 weeks of the establishment of the model, rats of the $\mathrm{UL}+\mathrm{CRSR}$ group were gavaged daily with CRSR $(6.67 \mathrm{~g} / \mathrm{kg})$. The serum concentrations of progesterone $(P)$ and estradiol $\left(\mathrm{E}_{2}\right)$ were determined by radioimmunoassay, the uterine index was measured by caliper measurement, and the pathological status was observed by hematoxylin and eosin stain. Gene expression profiling was checked by NimbleGen Rat Gene Expression Microarrays. The results indicated that the uterine mass of UL +CRSR rats was significantly shrunk and serum $P$ and $E_{2}$ levels significantly reduced compared to UL animals and nearly to the level of normal rats. Results of microarrays displayed the extensive inhibition of CRSR upon the expression of proliferation and deposition of extracellular matrix (ECM)-related genes, and significantly regulated a wide range of metabolism disorders. Furthermore, CRSR extensively regulated key pathways of the UL process, such as MAPK, PPAR, Notch, and TGF- $\beta /$ Smad. Regulation of the crucial pathways for the UL process and ECM metabolism may be the underlying mechanisms of CRSR treatment. Further studies will provide clear clues for effectively treating UL with CRSR.
\end{abstract}

Key words: Uterine leiomyoma; Transcriptional profiling; Extracellular matrix; Curcumae rhizoma; Sparganii rhizoma

\section{Introduction}

Uterine leiomyoma (UL, also called uterine fibroid) is a common, non-malignant, smooth muscle tumor, affecting 30 to $70 \%$ of females during their reproductive periods (1), having a tremendous medical and economic impact worldwide (2), costing approximately US\$ 5.9-34.4 billion annually (3). UL causes abnormal uterine bleeding, pelvic pain, bladder or bowel dysfunction, and subfertility. The proliferation of leiomyoma cells together with excessive deposition of extracellular matrix (ECM) is the crucial pathological process. These two mechanisms are tightly regulated by a complex network of interconnected pathways, including pathways of steroids, growth factors, TGF- $\beta /$ Smad, Wnt/ $\beta$-catenin, retinoic acid, vitamin $D$, and PPAR $\gamma(1,2,4)$.
Interestingly, aberrant signal transduction pathways in UL patients are not only intracellular, but also extracellular and intercellular pathways. Furthermore, partly due to the poor understanding of the underlying pathobiology, there is no effective medical treatment currently available $(1,4,5)$. Major therapy strategies are based on the inhibition of estrogen or progesterone, but UL tends to regrow once treatment is stopped. Therefore, understanding aberrations of these signaling pathways and their interconnections is critical for the development of satisfactory drugs.

Herbal preparations are commonly used as alternatives to surgical procedures. Yen and colleagues analyzed 35,786 diagnosed UL subjects in Taiwan, of whom $53.8 \%$

Correspondence: Cheng Peng: <pengchengchengdu@126.com> | Wei Jun Ding <dingwj123@163.com>

${ }^{*}$ These authors contributed equally to this work. 
had used Chinese herbal remedies (6). Jacoby et al. (7) reviewed that complementary and alternative treatments were extensively used in ULs, including exercise (45\%), diet $(34 \%)$, herbs $(37 \%)$, and acupuncture (16\%). Participants reported significant symptom improvement and few side effects with these interventions. Both clinical observations and animal research demonstrated that Chinese herbs can reduce the leiomyoma volume and shrink the size of the uterus. In fact, only 10 to $20 \%$ of UL patients require surgery; interventions such as traditional Chinese medicine (TCM) may eliminate the need for surgery in some cases, especially if treated early (8). TCM can also reduce the consumption of conventional chemical drugs (9).

Meta-analyses showed that Curcumae rhizoma and Sparganii rhizoma were among the frequently prescribed Chinese herbs for UL treatment (7-10). S. rhizoma exhibits anti-angiogenesis, anti-inflammation, and anti-estrogen effects (11-14). C. rhizoma is widely used in the therapy of UL but absolutely prohibited for the pregnant women in clinics (15-17). It possesses anti-inflammatory and anti-tumor activities and responds to multiple regulation of signaling pathways for ECM deposition (18), cell cycle arrest (19), and UL process (20). Moreover, TCM has long established the concise and synergistic effects of $C$. rhizoma and $S$. rhizoma, and, as a consequence, a compound with both was developed for UL therapies (21). Couplet medicines in TCM concept refer to two herbs that exhibit synergistic pharmaceutical and/or detoxic activities. C. rhizoma-S. rhizoma (CRSR) is a classical herb pair in clinics of TCM, and it has been used for thousands of years. ULs belong to the concept of "Zhengjia" and "Jiju", which are caused by "blood stasis" and "Qi stagnation" in TCM. CRSR is used for activating blood circulation and breaking the "Qi", and treating especially gynecologic tumors in TCM clinics (22).

Although CRSR possesses synergistic effects based on TCM theories in UL therapies, the underlying mechanisms are not clear. Here, we primarily verified the CRSR effect against UL based on the approach of transcriptional profiling.

\section{Material and Methods}

\section{Chemicals and reagents}

The dried herb of $C$. rhizoma was purchased from Sichuan Kelun Pharmaceutical Co. Ltd (China). The crude herb was crushed into powder and sieved by a 60-mesh sieve. According to the extraction method of volatile oil recorded in the Chinese Pharmacopoeia (2010 Edition), the volatile oil of $C$. rhizoma was extracted by steam distillation. Then, the volatile oil was collected for further use.

For preparation of the total flavonoids of S. rhizoma, the dried S. rhizoma was also purchased from Sichuan Kelun Pharmaceutical Co. Ltd. The crude herb was smashed into powder and sieved by a 60-mesh sieve. Total flavones were extracted with $10 \times$ volume of sample weight with $60 \%$ alcohol for three times, and $1.5 \mathrm{~h}$ each time. Finally, the above filtrates were combined, vacuum-dried to constant weight, and stored at $4^{\circ} \mathrm{C}$ for further analysis.

Diethylstilbestrol tablets (Cat No. 20100920, $0.5 \mathrm{mg} /$ tablet) were made by Hefei Jiu Lian Pharmaceutical Co., Ltd. (China). Progesterone for injection (Cat No. 120307, $10 \mathrm{mg} / \mathrm{mL}$ ) was made by Zhejiang Xian Ju Pharmaceutical Co., Ltd., China. The estradiol radioimmunoassay kit (Cat No. E10J015) and progesterone radioimmunoassay kit (Cat No. E10J023) were purchased from Beijing Fu Rui Biological Engineering Co., Ltd., China. DAB color kit (Cat No. 12182A01), horseradish peroxidase-labeled streptavidin working solution (Cat No. 12122A08) and citrate buffer ( $\mathrm{pH} \mathrm{6.0,} \mathrm{Cat} \mathrm{No.} \mathrm{ZLI-9065)} \mathrm{were} \mathrm{purchased} \mathrm{from}$ Beijing Zhong Shan Golden Bridge Biotechnology Co., Ltd., China.

\section{Animal model and herbal treatment}

Thirty female Sprague Dawley rats, weighing $200 \pm 20 \mathrm{~g}$, were obtained from the Experimental Animal Center, Chengdu University of TCM (China). After adaptive feeding for 1 week, rats were randomly divided into 3 groups: normal control (NC, $n=10)$, UL $(n=8)$, and CRSR therapy $(U L+C R S R, n=10)$. All experiments were approved by the Institutional Animal Care and Use Committee of Chengdu University of TCM. Rats of both UL + CRSR and UL groups received diethylstilbestrol $(0.167 \mathrm{mg} / \mathrm{kg})$ by gavage every other day, and intramuscular injection of progesterone $(1 \mathrm{mg} / \mathrm{kg})$ once a week, for 20 weeks. Animals of the NC group were maintained in the same conditions without any treatment. From 11 weeks of modelinduction, rats of the UL + CRSR group were gavaged daily with the volatile oil of $C$. rhizoma and the total flavonoids of $S$. rhizoma $(6.67 \mathrm{~g} / \mathrm{kg}), 10$ times the clinical dosage for human beings (w/w), which was calculated based on the crude herbs used for the extractions. Animals were observed daily in order to record any posttreatment complication or adverse event.

\section{Sample collection}

At the end of the 20-week experiment, animals were anesthetized with ether and the following procedures were conducted. Blood sample was collected from the femoral artery and centrifuged at $1048 \mathrm{~g}$ and $5^{\circ} \mathrm{C}$ for $15 \mathrm{~min}$. The serum was collected and the concentrations of progesterone $(P)$ and estradiol $\left(E_{2}\right)$ were immediately determined by radioimmunoassay kits, in accordance with the supplier's instruction. Length and root diameter of the uterine horn were measured by caliper measurement. The uterine coefficient was calculated using the formula: uterine coefficient $=$ uterine weight $(\mathrm{mg}) /$ rat body weight $(\mathrm{g}) \times 100 \%$. UL tissues were collected from all rats in areas adjacent to the leiomyoma and from the endometrial, intramural, and serosal layers at a location distant from the leiomyoma within the uterus. In the normal control animals, 
tissue samples at the same areas were collected. Uterine tissues were divided into two parts: one was immediately preserved in liquid nitrogen and then stored at $-80^{\circ} \mathrm{C}$ for transcriptional analysis and the other was formalin-fixed for 15-20 h, paraffin-embedded, and subjected to hematoxylin and eosin (H\&E) histological observation.

\section{Transcriptional profiling analysis}

Gene expression profiling analysis was performed using NimbleGen Rat Gene Expression Microarrays (Roche NimbleGen, USA) (135 K arrays covering 26,419 UniGenes collected from NCBI). Total RNA from each sample was quantified by a NanoDrop ND-1000 (Thermo, USA) and RNA integrity was assessed by standard denaturing agar gel electrophoresis (Biowest Agarose, Spain). Five micrograms of total RNA of each sample was utilized for labeling and array hybridization with the following steps: 1) reverse transcription by the Invitrogen Superscript ds-cDNA synthesis kit (USA); 2) ds-cDNA labeling with NimbleGen one-color DNA labeling kit (Roche NimbleGen, USA); 3) array hybridization using the NimbleGen Hybridization System followed by washing with the NimbleGen wash buffer kit; 4) array scanning using the Axon GenePix 4000B microarray scanner (Molecular Devices Corporation, USA). Scanned images were then imported into NimbleScan software (version 2.5) for grid alignment and expression data analysis. Expression data were normalized through quantile normalization and the Robust Multichip Average (RMA) algorithm included in the NimbleScan software. The Probe level ( ${ }^{*}$ norm_RMA.pair) files and Gene level (*_RMA.calls) files were generated after normalization. The gene data were imported into Agilent GeneSpring GX software (USA, version 11.5.1) for further analysis. Genes that had values greater than or equal to the cut-off of 100.0 were chosen for data analysis. Microarray data could be shared by relevant experts for academic research upon request. Differentially expressed genes were identified through fold-change filtering. Pathway analysis and Gene Ontology (GO) analysis (http://geneontology.org/) were applied to determine the roles of these differently expressed genes in biological pathways or GO terms. Finally, hierarchical clustering was performed to show different gene expression profiling among samples.

Genes that were found to be significantly different in a pairwise comparison were further analyzed for canonical pathways, networks, transcription factors, and biological functions using the Ingenuity Pathway Analysis (IPA) software (Ingenuity Systems, USA). The IPA software is based on computational algorithms of the connectivity from information obtained within the IPA database. IPA analysis accounts for the type of chip used with a score assigned to rank networks according to their relevance to the gene list provided. Canonical pathways were determined by analyzing a ratio of the number of genes that map to the pathway, divided by the total number of genes in the pathway that are represented by the chip probes, and the $P$ value was calculated by Fisher's exact test to determine the probability that the association was due to chance alone.

\section{Statistical analysis}

The statistical software SPSS 16.0 was used to carry out all data analysis. Results of quantitative experiments are reported as means \pm SD. Each data point represents the average of three independent experiments. One-way analysis of variance was used for multiple group comparisons. When only two groups were compared, the Student's unpaired $t$-test was used. Results were considered statistically significant if the $P$ value was less than 0.05 .

\section{Results}

\section{CRSR significantly reduced UL parameters}

Two representative indexes of uterine size (i.e., the length of the uterine horn and the root diameter of the uterine horn) were both significantly reduced in UL + CRSR rats $(P<0.01)$. On the other hand, there was no significant difference in the uterine size of UL + CRSR rats compared with those of the NC animals, yet some increase of the uterus was still observed after CRSR treatment (Figure 1A). The uterine coefficient in UL increased compared to NC. The uterine coefficient in UL + CRSR significantly decreased compared with UL (Figure 1B). The uterine coefficient did not differ significantly between NC and UL + CRSR $(P>0.05)$.

\section{Histological changes in the uterus}

Representative H\&E micrographs from NC, UL, and $\mathrm{UL}+\mathrm{CRSR}$ rats are shown in Figure 2. Fibroid sections from UL rats showed distorted, sparsely distributed nuclei with smudged chromatin pattern, and ill-defined nuclear and cytoplasm outline (Figure 2B). Major histological changes in fibroid sections from the UL + CRSR rats were mild hypertrophy and focal mild hyperplasia of smooth muscle cells, yet neatly arranged (Figure 2C). Conversely, uterine samples from NC rats showed the typical interlacing bundles of smooth muscle cells (Figure 2A). The smooth muscle cells were elongated and spindleshaped, and had indistinct cell borders and abundant pale eosinophilic cytoplasm. The nuclei were uniform, crowded, and tightly packed with a fine granular chromatin pattern. Based on 200 representative cells from H\&E micrographs derived from each group, the average ECM thickness of NC, UL, and UL + CRSR was $12.64 \pm 3.87$, $21.57 \pm 5.35$, and $14.24 \pm 3.49 \mu \mathrm{m}$, respectively. Hence, histological uterine changes confirmed the therapeutic effect of CRSR.

\section{CRSR markedly reduced the concentrations of serum $E_{2}$ and $P$}

High levels of serum $E_{2}$ and $P$ are key factors for the promotion of the UL process. We observed by standard radioimmunoassay that UL rats had significantly higher 

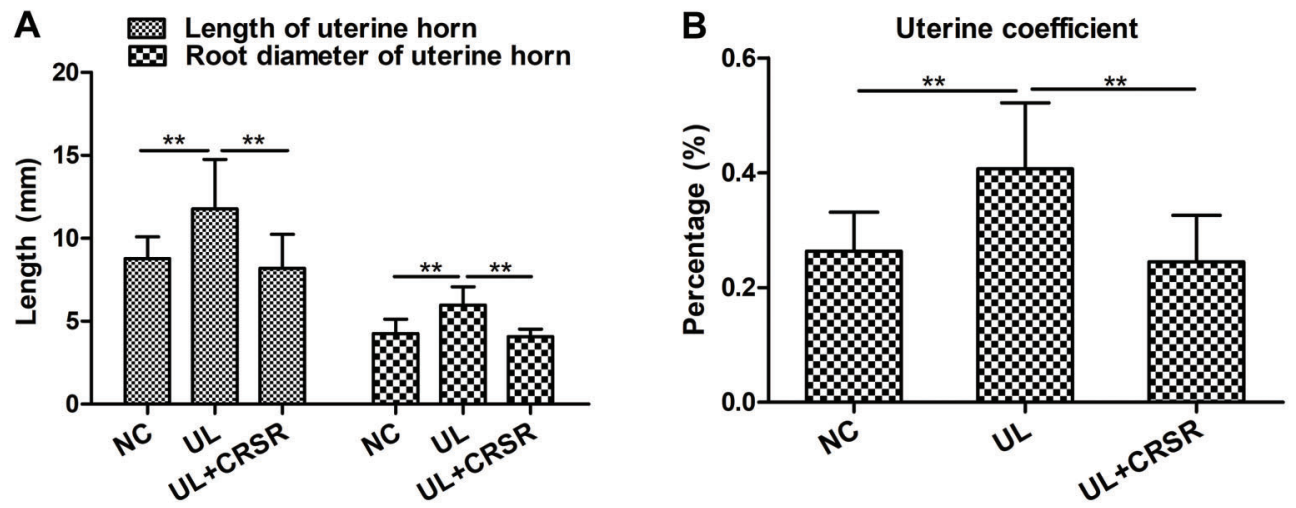

Figure 1. Effects of Curcumae rhizoma-Sparganii rhizoma (CRSR) treatment on the length of the uterine horn, the root diameter of the uterine horn $(\mathbf{A})$, and the uterine coefficient $(\mathbf{B})$. Data are reported as means \pm SD. NC: normal control, $n=10$; UL: uterine leiomyomas, $n=8$; UL + CRSR: treatment group, $n=10 .{ }^{* *} \mathrm{P}<0.01$ compared with the UL model (ANOVA).
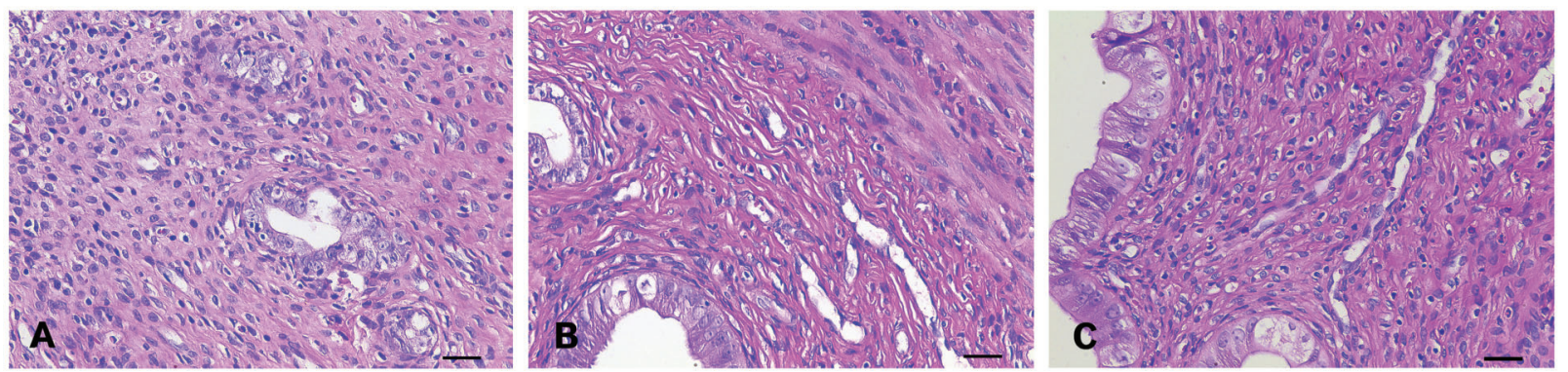

Figure 2. Representative micrographs obtained by hematoxylin and eosin stain. The original magnification was $400 \times$. A: NC, normal control; B: UL, uterine leiomyomas; C: UL + CRSR, treatment group with Curcumae rhizoma-Sparganii rhizoma. Magnification bar: $30 \mu \mathrm{m}$.

A

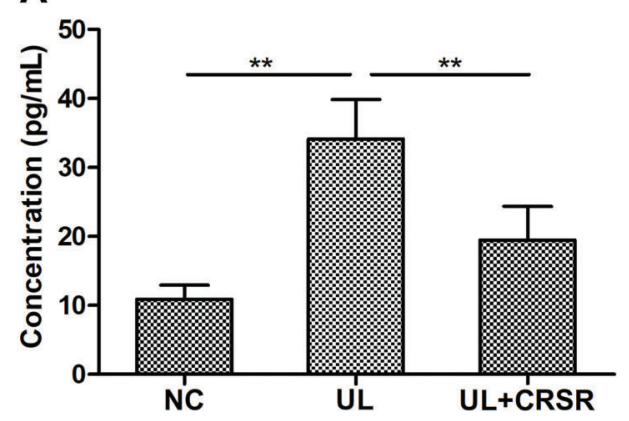

B

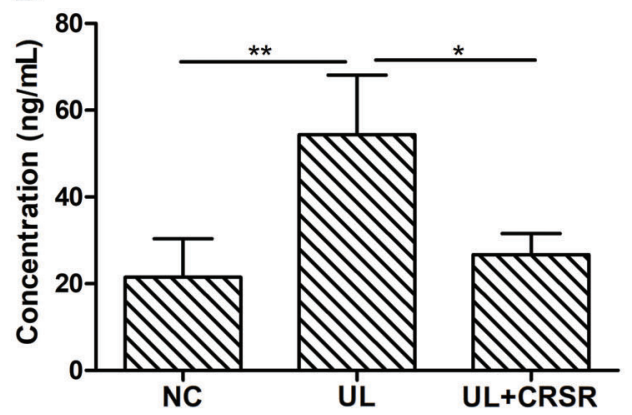

Figure 3. Effects of Curcumae rhizoma-Sparganii rhizoma (CRSR) treatment on the key hormones of uterine leiomyomas (UL): A, estradiol $\left(E_{2}\right)$; $B$, progesterone $(P)$. Data are reported as means $\pm S D$. NC: normal control, $n=10$; UL: uterine leiomyomas, $n=8$; $\mathrm{UL}+$ CRSR: treatment group, $n=10$. ${ }^{*} \mathrm{P}<0.05$, ${ }^{*} \mathrm{P}<0.01$ (ANOVA).

levels of serum $E_{2}$ and $P$, compared with $\mathrm{NC}$ rats. After the CRSR treatment for 10 weeks, both hormones were restored almost to that of the NC animals (Figure 3). Therefore, these data suggest that CRSR reduced the serum hormones that otherwise can promote UL development, and subsequently led to shrinkage of ULs.

\section{CRSR inhibited the expression of proliferation- and ECM-related genes}

ULs are characterized by an abundance of ECM, which is mainly composed of collagen and fibronectin (9). Previous reports have demonstrated that genes that encode ECM proteins were expressed abnormally in leiomyomas. 
Our microarray results demonstrated a large number of differently expressed genes with roles in signal transduction pathways, metabolism, cell proliferation, and ECM formation (complete data not shown). Table 1 lists the top ten down- or up-regulated genes. For instance, lipocalin 2 is associated with multiple biological processes such as pheromone transport, immune response, retinoid binding, and MMP-9 synthesis (23); aquaporin 8 is a small integral membrane protein related to ECM formation (24); the classic role of oxytocin receptor is as an inducer of uterine contractions (25). Compared with NC rats, genes of lipocalin 2 and aquaporin 8 were extremely up-regulated in UL animals, suggesting the over-expression of ECM in UL rats. These results imply that CRSR might reduce uterine size in UL animals by adjusting the ECM-associated genes and their regulation pathways (Table 1 ).

GO analysis more clearly reflected the primary patterns of the differently expressed genes between NC, UL, and
UL + CRSR animals (Table 2). Results of GO biological process classification displayed that the down-regulated response to stimulus and up-regulated metabolic process were the most distinguished features between UL and NC rats. The metabolic process, biosynthesis process, response to stimulus, and developmental process were quite different between UL + CRSR and UL animals. As for UL + CRSR and NC groups, metabolic process, biological regulation, regulation of cellular and biological process, multi-cellular organism development, and cellular process exhibited differences. These results indicated that in biological process, regulation of metabolism and development, response to stimulus, and adjustment of biosynthesis process were majorly responsive for the pathological process of UL. Such conditions were also related to the pharmacological effects of CRSR.

Results of GO cellular component classification demonstrated that the down-regulated genes were majorly involved

Table 1. Top ten significantly regulated genes between different groups.

\begin{tabular}{|c|c|c|c|c|}
\hline & Gene symbol & Fold change & Gene symbol & Fold change \\
\hline \multirow[t]{10}{*}{ NC vs UL } & Lcn2 & 69.817 & Slc39a1 & -9.578 \\
\hline & Gal & 40.528 & Nov & -8.501 \\
\hline & Aqp8 & 35.497 & LOC682601 & -7.880 \\
\hline & Ngfr & 33.604 & Lrrc17 & -6.492 \\
\hline & Cbln1 & 32.587 & Lrrc27 & -6.108 \\
\hline & Rasd1 & 27.603 & Spon2 & -6.083 \\
\hline & Ccl7 & 18.854 & Hmgcs2 & -6.057 \\
\hline & Oxtr & 17.999 & Serpina3n & -6.049 \\
\hline & Gadd45g & 14.546 & Ctla2a & -5.660 \\
\hline & LOC688246 & 12.186 & Tppp & -5.144 \\
\hline \multirow[t]{10}{*}{ UL + CRSR vs UL } & Slc39a1 & 15.484 & Nxt1 & -15.458 \\
\hline & Serpina3n & 12.126 & LOC688976 & -4.128 \\
\hline & Hmgcs2 & 4.784 & LOC680511 & -3.998 \\
\hline & Cxcl6 & 4.650 & Tpcn2 & -3.987 \\
\hline & II13ra2 & 3.105 & LOC685415 & -3.646 \\
\hline & Ccr7 & 3.079 & RGD1560815 & -3.613 \\
\hline & Adipoq & 3.008 & LOC502486 & -3.608 \\
\hline & Wfdc10 & 2.995 & LOC499487 & -3.546 \\
\hline & LOC688556 & 2.860 & LOC684897 & -3.416 \\
\hline & Snip & 2.852 & LOC684285 & -3.395 \\
\hline \multirow[t]{10}{*}{$\mathrm{UL}+\mathrm{CRSR}$ vs NC } & Lcn2 & 57.935 & Nxt1 & -12.546 \\
\hline & Ngfr & 45.672 & Nov & -8.293 \\
\hline & Aqp8 & 35.914 & LOC682601 & -7.454 \\
\hline & Cbln1 & 30.296 & LOC690062 & -6.763 \\
\hline & Rasd1 & 29.853 & Spon2 & -6.404 \\
\hline & Gal & 19.618 & Trpv6 & -6.366 \\
\hline & Oxtr & 17.296 & Lrrc17 & -5.744 \\
\hline & Gadd45g & 15.439 & Ctla2a & -5.743 \\
\hline & Gpr88 & 11.130 & Cpm & -5.725 \\
\hline & Ccl7 & 11.077 & LOC685369 & -5.383 \\
\hline
\end{tabular}

The positive and negative numbers indicate up- and down-regulated genes, respectively. NC: normal control; UL: uterine leiomyomas; UL + CRSR: treatment group with a traditional herb pair, Curcumae rhizoma and Sparganii rhizoma. 
Table 2. Gene ontology (GO) analysis of the differently expressed genes.

\begin{tabular}{|c|c|c|c|}
\hline & UL vs NC & UL + CRSR vs UL & UL + CRSR vs NC \\
\hline \multicolumn{4}{|l|}{ Biological Process } \\
\hline & Response to stimulus & Metabolic process & Metabolic process \\
\hline & Multicellular organism process & Biosynthetic process & Multicellular organism development \\
\hline & Primary metabolic process & Regulation of biological process & $\begin{array}{c}\text { Cellular component organization } \\
\text { or biogenesis }\end{array}$ \\
\hline & Metabolic process & Response to stimulus & Location \\
\hline & Anatomical structure development & Developmental process & Anatomic structure development \\
\hline & Multicellular organism development & $\begin{array}{c}\text { Multicellular organism } \\
\text { process }\end{array}$ & Regulation of biological process \\
\hline & Cellular process & Protein metabolic process & Biological regulation \\
\hline & Localization & & Cellular process \\
\hline & Regulation of biological process & & Regulation of cellular process \\
\hline \multicolumn{4}{|l|}{ Cellular component } \\
\hline & Extracellular region & Cell & Organelle \\
\hline & Extracellular matrix & Intracellular & Extracellular region \\
\hline & Neuron projection & organelle & Extracellular matrix \\
\hline & Axon & Intracellular organelle & Ribosome \\
\hline & Perinuclear region & Cytoplasm & Ribonucleoprotein \\
\hline & Intermediate filament & Extracellular region & Intermediate filament \\
\hline & Organelle & Plasma membrane & Keratin filament \\
\hline & Intracellular & Extracellular matrix & Cytoplasm \\
\hline & Cytoplasm & Cell periphery & Intracellular \\
\hline \multicolumn{4}{|l|}{ Molecular Function } \\
\hline & Binding & Protein binding & Binding \\
\hline & Protein binding & Structure of ribosome & Receptor binding \\
\hline & Catalytic activity & Structural molecular activity & Structural molecular activity \\
\hline & Metal ion binding & Microtube binding & DNA binding \\
\hline & Small molecular bind & Glucose binding & Small molecular bind \\
\hline & Nucleotide binding & Enzyme binding & Structure of ribosome \\
\hline & Receptor binding & Magnesium ion binding & Metal ion binding \\
\hline & Transferase activity & Apolipoprotein binding & Protein binding \\
\hline & Cytokine activity & Peptidase regulator activity & Cytokine activity \\
\hline & Hormone activity & Neurotransmitter receptor & Catalytic activity \\
\hline
\end{tabular}

NC: normal control; UL: uterine leiomyomas; UL + CRSR: treatment group conducted with a traditional herb pair, Curcumae rhizoma and Sparganii rhizoma.

in the extra-cellular region or matrix, and up-regulated intracellular components were the most distinguished features between UL and NC animals. This indicates the abnormal metabolism of critical components was involved in the UL process. Interestingly, these abnormal metabolisms were substantially reversed in the UL +CRSR animals. Comparing UL + CRSR with NC rats, any of the extra- and intra-cellular components were adjusted, and constitution of ECM, such as keratin filaments, intermediate filament, and ribonucleoprotein, was also intensively regulated (Table 2 ).

Results of the GO molecular function classification showed that diverse binding functions (i.e., protein, metal ion, small molecular, nucleotide, and receptor binding) and various metabolic or immunological activities were among the differences between NC and UL animals. Comparing $\mathrm{UL}+\mathrm{CRSR}$ with NC rats, adjustment of various binding functions were found, suggesting the recovered activities of diverse binding functions were related to the therapy effects of CRSR against UL process. Comparing UL + CRSR with UL rats, similar results were observed for a series of binding functions and catalytic and cytokine activities (Table 2).

\section{CRSR extensively regulated the key signal pathways of the UL process}

The top five significant canonical pathways identified by IPA are displayed at Table 3. Nine of the down-regulated pathways were identified in the UL rats, compared to NC rats (Supplementary Table S1). These are metabolism 
Table 3. Top five differentially expressed pathways identified by Ingenuity Pathway Analysis.

\begin{tabular}{|c|c|c|c|c|c|c|}
\hline & \multicolumn{3}{|c|}{ Down-regulated pathway } & \multicolumn{3}{|c|}{ Up-regulated pathway } \\
\hline & Pathway Name & $\begin{array}{c}\mathrm{P} \\
\text { value }\end{array}$ & $\begin{array}{l}\text { Enrichment } \\
\text { Score }\end{array}$ & Pathway Name & $\begin{array}{c}\mathrm{P} \\
\text { value }\end{array}$ & $\begin{array}{c}\text { Enrichment } \\
\text { Score }\end{array}$ \\
\hline \multirow[t]{5}{*}{ NC vs UL } & $\begin{array}{l}\text { Pantothenate and CoA } \\
\text { biosynthesis }\end{array}$ & 0.001 & 2.964 & Hedgehog signaling pathway & 0.049 & 1.314 \\
\hline & Arachidonic acid metabolism & 0.004 & 2.364 & Purine metabolism & 0.045 & 1.350 \\
\hline & Drug metabolism - cytochrome P450 & 0.005 & 2.298 & Seleno-compound metabolism & 0.043 & 1.363 \\
\hline & Glutathione metabolism & 0.006 & 2.243 & RNA degradation & 0.041 & 1.385 \\
\hline & $\begin{array}{l}\text { Metabolism of xenobiotics by } \\
\text { cytochrome P450 }\end{array}$ & 0.006 & 2.213 & $\begin{array}{l}\text { Cysteine and methionine } \\
\text { metabolism }\end{array}$ & 0.033 & 1.486 \\
\hline \multirow[t]{5}{*}{ UL + CRSR vs UL } & Influenza A & 0.001 & 2.884 & PPAR signaling pathway & 0.046 & 1.335 \\
\hline & Graft vs host disease & 0.014 & 1.865 & Chemokine signaling pathway & 0.043 & 1.365 \\
\hline & Allograft rejection & 0.017 & 1.778 & $\begin{array}{c}\text { Adipocytokine signaling } \\
\text { pathway }\end{array}$ & 0.004 & 2.453 \\
\hline & Type I diabetes mellitus & 0.018 & 1.755 & $\begin{array}{l}\text { Pantothenate and CoA } \\
\text { biosynthesis }\end{array}$ & 0.002 & 2.660 \\
\hline & Gastric acid secretion & 0.018 & 1.733 & & & \\
\hline \multirow[t]{5}{*}{ UL + CRSR vs NC } & Arachidonic acid metabolism & 0.001 & 3.066 & Purine metabolism & 0.048 & 1.315 \\
\hline & $\begin{array}{l}\text { Antigen processing and } \\
\text { presentation }\end{array}$ & 0.002 & 2.757 & RNA transport & 0.042 & 1.381 \\
\hline & Drug metabolism - cytochrome P450 & 0.006 & 2.220 & Seleno-compound metabolism & 0.027 & 1.575 \\
\hline & Cell adhesion molecules & 0.006 & 2.208 & TGF-beta signaling pathway & 0.024 & 1.613 \\
\hline & Serotonergic synapse & 0.009 & 2.032 & Pyrimidine metabolism & 0.023 & 1.643 \\
\hline
\end{tabular}

NC: normal control; UL: uterine leiomyomas; UL+CRSR: treatment group conducted with an herb pair, Curcumae rhizoma and Sparganii rhizoma.

pathways, i.e., metabolism of cofactors, vitamins, xenobiotics, amino acids, and lipids. On the other hand, 28 upregulated pathways were identified from the uterus tissues of UL rats (Supplementary Table S2), which are mainly involved in infectious diseases, nucleotide and amino acid metabolisms, signal transduction, ECM metabolism, and regulation of the endocrine-immune-nervous web. This indicates that the UL process is involved with abnormal metabolism, excessive ECM synthesis, and disturbed signal transduction and immune function (26).

Fewer differently expressed pathways were detected between UL + CRSR and UL rats (Table 3 and Supplementary Table S3). The down-regulated pathways belonged to diseases (metabolic, digestive, immunological, or infectious diseases), and attenuated functions of antigen processing and presentation. Four up-regulated pathways were involved in pantothenate and CoA biosynthesis, PPAR, adipocytokine, and chemokine signaling. Results indicated that only limited pathways were recovered to normal status.

There were sixteen down-regulated pathways between UL + CRSR and NC rats (Supplementary Table S4). These pathways are related to metabolism (lipids, xenobiotics, and amino acids), immune system and metabolic diseases, ECM formation, and systemic regulation. We also identified 25 up-regulated pathways in UL + CRSR animals (Supplementary Table S5). These pathways are mainly related to diseases (metabolic or infectious diseases), nucleotide and amino acids metabolism, signal transductions (such as MAPK, Notch, and TGF-beta pathways), ECM metabolism, and regulation of the endocrineimmune-nervous system. These results suggested that there were still notable differences between UL + CRSR and NC rats, particularly in ECM metabolism, immune functions, and signal transduction pathways involved in fibroid formation.

Taken together, our results demonstrated that CRSR treatment can extensively and profoundly modify the signaling pathways involved in UL development and remedy processes. These include at least three approaches: 1) suppressing ECM metabolism directly; 2) regulating key signaling of UL process, such as MAPK, PPAR, Notch, and TGF-beta signaling pathways; 3) tuning signaling pathways of global metabolism and immunity that indirectly regulate the development of UL process.

\section{Discussion}

$\mathrm{UL}$ is a complex disease that exerts a huge burden on healthcare resources worldwide. The management of UL 
is an enormous challenge given the high incidence and the lack of an effective and safe nonsurgical treatment $(2,4,5,6)$. Although hysterectomy remains the main option for the treatment of UL, the surgical approach is not always a favorable choice, particularly in women who desire to preserve their fertility. Therefore, the development of an effective and nonsurgical therapeutic strategy is a critical need in female health care.

Chinese herbs have been extensively used in oriental countries, showing advantages in treating gynecologic disorders (6-11). Meta-analyses show that CRSR was frequently used for effective therapy of UL (12-17). High concentrations of Grailsine-Al-glycoside, the bioactive component of $S$. rhizoma, strongly suppressed cell proliferation in a dose-dependent fashion in A549, MCF-7, HepG2, and HeLa cells (13). Sparstolonin B, a bioactive compound isolated from S. rhizoma, inhibited endothelial cell tube formation and cell migration in part by downregulating $\mathrm{CCNE} 2$ and $\mathrm{CDC} 6$, halting progression through the $\mathrm{G} 1 / \mathrm{S}$ checkpoint (14). Sparstolonin B achieved antiinflammation effects by the attenuation of LPS-induced phosphorylation of signaling molecules Erk1/2 and Akt (15). On the other hand, C. rhizoma is also widely used in the therapy of ULs (16). The essential oil of C. rhizoma presented anti-angiogenic activity in vitro and in vivo, resulting in the suppression of tumor growth and metastasis, by inhibiting the phosphorylation of ERK1/2 and $\mathrm{AKT} / \mathrm{NF}-\mathrm{KB}$ signaling pathways, and enhancing the phosphorylation of JNK1/2 and p38 (16-18). Petroleum ether extracts of $C$. rhizoma produce a significant $\mathrm{G} 0 / \mathrm{G} 1$ cell cycle arrest at the concentration of $300 \mu \mathrm{g} / \mathrm{mL}$ (19). Curcumenol, a sesquiterpene isolated from C. rhizoma, possess anti-inflammatory and anti-tumor activities by inhibiting Akt-dependent NF- $\mathrm{BB}$ and p38-MAPK signaling (20). The chloroform-fraction of C. rhizoma significantly inhibited leiomyoma cell proliferation (21). However, the synergistic effects of $C$. rhizoma and $S$. rhizoma against UL growth have not been studied. Therefore, we explored the anti-fibroid effects of CRSR through a novel approach, transcriptional profiling.

The process of UL development is apparently regulated by the serum levels of sex hormones. Both estrogen and progesterone play crucial roles in UL growth $(4,5,16)$. We observed significantly reduced serum concentrations of both estrogen and progesterone in CRSR-treated rats, indicating the pharmaceutical implication of CRSR (12-15). Additionally, CRSR therapy can markedly reduce uterine size represented by the length of the under point of uterine horn, the root diameter of uterine horn points, and uterine coefficient, and substantially recover the pathological conditions. These results indicated that the therapy effects of CRSR achieved the same levels as certain famous TCM formula, such as Gui-zhi Fu-Ling decoction (11), yet fewer components were recruited. Furthermore, histological observation reconfirmed that CRSR can effectively attenuate the process of ECM deposition and UL growth. Results of both pathology and endocrine assays indicated that CRSR can inhibit UL growth.

To further understand the potential mechanisms of UL shrinkage induced by CRSR, we analyzed the transcriptome features by cDNA microarrays. ULs are characterized by the excess synthesis and abundant accumulation of ECM proteins $(1,2,4,5,27)$. Our microarray results demonstrated a large number of abnormally expressed genes that were mainly involved in cell proliferation and ECM formation, metabolism, and signal transduction pathways, suggesting that CRSR might reduce the UL process by intricately tuning the ECM-associated genes and/or their regulation pathways. Furthermore, $\mathrm{GO}$ analysis of these abnormally expressed genes clarified the regulation patterns among NC, UL, and UL + CRSR animals. Results of $\mathrm{GO}$ biological process demonstrated that global adjustment of metabolic, biosynthetic, and developmental processes, promotion of the response to stimulus, and inhibition of the ECM formation were the key points of CRSR treatment. Results of GO cellular component classification demonstrated that the UL + CRSR group directly reversed the abnormal metabolism of UL process, especially attenuating the extracellular matrix. Results of GO molecular function classification showed that diverse binding functions and various metabolic and immunological activities were disturbed in UL rats, while CRSR recovered some important disorders of binding functions, and immunological and metabolic activities (28-30). Collectively, the molecular mechanisms of CRSR against the UL process are focused on the arrestment of cell proliferation and ECM-formation genes, intensively tuning a wide range of metabolism disorders.

Results of signaling pathways analysis showed that CRSR can delicately and extensively tune the UL processes. The ECM in UL animals is not only excessive, but also has alterations in its composition (31). The disordered ECM represents the major sign of UL patients $(27,31)$. Altered ECM and its regulating signaling in UL are novel targets for the development of pharmaceutical agents $(28,32)$. Also, excessive ECM directly disturbs extraand inter-cellular signaling networks (1,2,4,5,33-35). Our results demonstrated four characteristics of CRSR against UL: a) delicately regulated crucial signaling pathways of the UL process, such as PPAR, MAPK, TGFbeta/Smad, and Notch signaling pathways; b) extensively regulated the signaling pathways related to diverse metabolisms, especially xenobiotics biodegradation and metabolism, metabolism of cofactors and vitamins, nucleotide and amino acids metabolism; c) directly suppressed the ECM metabolism; and d) globally impacted the UL process by tuning the endocrine-immune-nervous system.

Our findings shed light on the effective treatment of UL, and provided new insights into the mechanisms 
underlying the effects of CRSR against the UL process.

\section{Supplementary material}

Click here to view [pdf].

\section{References}

1. Borahay MA, Al-Hendy A, Kilic GS, Boehning D. Signaling pathways in leiomyoma: understanding pathobiology and implications for therapy. Mol Med 2015; 21: 242-256, doi: 10.2119/molmed.2014.00053.

2. Davis BJ, Risinger JI, Chandramouli GV, Bushel PR, Baird $\mathrm{DD}$, Peddada SD. Gene expression in uterine leiomyoma from tumors likely to be growing (from black women over 35) and tumors likely to be non-growing (from white women over 35). PLoS One 2013; 8: e63909, doi: 10.1371/journal.pone. 0063909.

3. Mauskopf J, Flynn M, Thieda P, Spalding J, Duchane J. The economic impact of uterine fibroids in the United States: a summary of published estimates. J Womens Health 2005; 14: 692-703, doi: 10.1089/jwh.2005.14.692.

4. Shen $Y, W u Y, L u Q$, Zhang $P$, Ren M. Transforming growth factor- $\beta$ signaling pathway cross-talking with $E R \alpha$ signaling pathway on regulating the growth of uterine leiomyoma activated by phenolic environmental estrogens in vitro. Tumour Biol 2016; 37: 455-462, doi: 10.1007/s13277-0153813-4.

5. Bulun SE, Moravek MB, Yin P, Ono M, Coon JS5th, Dyson MT, et al. Uterine leiomyoma stem cells: linking progesterone to growth. Semin Reprod Med 2015; 33: 357-365, doi: 10.1055/s-0035-1558451.

6. Yen HR, Chen YY, Huang TP, Chang TT, Tsao JY, Chen BC, et al. Prescription patterns of Chinese herbal products for patients with uterine leiomyoma in Taiwan: A nationwide population-based study. J Ethnopharmacol 2015; 171: 223230, doi: 10.1016/j.jep.2015.05.038.

7. Jacoby VL, Jacoby A, Learman LA, Schembri M, Gregorich $\mathrm{SE}$, Jackson R, et al. Use of medical, surgical and complementary treatments among women with fibroids. Eur $J$ Obstet Gynecol Reprod Biol 2014; 182: 220-225, doi: 10.1016/j.ejogrb.2014.09.004.

8. Zhou J, Qu F. Treating gynaecological disorders with traditional Chinese medicine: a review. Afr J Tradit Complement Altern Med 2009; 6: 494-517, doi: 10.4314/ajtcam. v6i4.57181.

9. Su SY, Muo $\mathrm{CH}$, Morisky DE. Use of Chinese medicine correlates negatively with the consumption of conventional medicine and medical cost in patients with uterine leiomyomas: a population-based retrospective cohort study in Taiwan. BMC Complement Altern Med 2015; 15: 129-136, doi: 10.1186/s12906-015-0645-0.

10. Liu JP, Yang H, Xia Y, Cardini F. Herbal preparations for uterine leiomyomas. Cochrane Database Syst Rev 2013; 4: CD005292-7, doi: 10.1002/14651858.CD005292.pub3.

11. Chen NN, Han M, Yang H, Yang GY, Wang YY, Wu XK, et al. Chinese herbal medicine Guizhi Fuling Formula for treatment of uterine leiomyomas: a systematic review of randomised

\section{Acknowledgments}

This research was supported by the National Natural Science Foundation of China (NSFC, Grant No. 81001668 and 81673878) and Chinese Scholarship Council (CSC, Grant No. 201708510029).

clinical trials. BMC Complement Altern Med 2014; 14: 2-6, doi: 10.1186/1472-6882-14-2.

12. Sun J, Wang S, Wei YH. Reproductive toxicity of Rhizoma sparganii (Sparganium stoloniferum Buch.-Ham.) in mice: mechanisms of anti-angiogenesis and anti-estrogen pharmacologic activities. J Ethnopharmacol 2011; 137: 14981503, doi: 10.1016/j.jep.2011.08.026.

13. Zhang JW, Wei YH. Anti-cancer effects of Grailsine-Alglycoside isolated from Rhizoma sparganii. BMC Complement Altern Med 2014; 14: 82-86, doi: 10.1186/1472-688214-82.

14. Bateman HR, Liang $Q$, Fan $D$, Rodrigue $V$, Lessner SM. Sparstolonin B inhibits pro-angiogenic functions and blocks cell cycle progression in endothelial cells. PLoS One 2013; 8: e70500, doi: 10.1371/journal.pone.0070500.

15. Liang Q, Yu F, Cui X, Duan J, Wu Q, Nagarkatti P, et al. Sparstolonin $B$ suppresses lipopolysaccharide-induced inflammation in human umbilical vein endothelial cells. Arch Pharm Res 2013; 36: 890-896, doi: 10.1007/s12272-013-0120-8.

16. Chen W, Lu Y, Gao M, Wu J, Wang A, Shi R. Antiangiogenesis effect of essential oil from Curcuma zedoaria in vitro and in vivo. J Ethnopharmacol 2011; 133: 220-226, doi: 10.1016/j.jep.2010.09.031.

17. Chen CC, Chen Y, Hsi YT, Chang CS, Huang LF, Ho CT, et al. Chemical constituents and anticancer activity of Curcuma zedoaria roscoe essential oil against non-small cell lung carcinoma cells in vitro and in vivo. J Agric Food Chem 2013; 61: 11418-11427, doi: 10.1021/jf4026184.

18. Zhou Y, Shen J, Xia L, Wang Y. Curcuma zedoaria (Berg.) Rosc. essential oil and paclitaxel synergistically enhance the apoptosis of SKOV3 cells. Mol Med Rep 2015; 12: 1253-1257, doi: 10.3892/mmr.2015.3473.

19. Gao XF, Li QL, Li HL, Zhang HY, Su JY, Wang B, et al. Extracts from Curcuma zedoaria inhibit proliferation of human breast cancer cell MDA-MB-231 in vitro. Evid Based Complement Alternat Med 2014; 2014: 730678, doi: 10.1155/ 2014/730678.

20. Lo JY, Kamarudin MN, Hamdi OA, Awang K, Kadir HA. Curcumenol isolated from Curcuma zedoaria suppresses Akt-mediated NF-KB activation and p38 MAPK signaling pathway in LPS-stimULated BV-2 microglial cells. Food Funct 2015; 6: 3550-3559, doi: 10.1039/C5FO00607D.

21. Bajracharya P, Lee EJ, Lee DM, Shim SH, Kim KJ, Lee SH, et al. Effect of different ingredients in traditional Korean medicine for human uterine leiomyoma on normal myometrial and leiomyomal smooth muscle cell proliferation. Arch Pharm Res 2009; 32: 1555-1563, doi: 10.1007/s12272-0092107-z.

22. Xu GL, Geng D, Xie M, Teng KY, Tian YX, Liu ZZ, et al. Chemical composition, antioxidative and anticancer activities 
of the essential oil: Curcumae rhizoma-Sparganii rhizoma, a traditional herb pair. Molecules 2015; 20: 15781-15796, doi: 10.3390/molecules200915781.

23. Chamero P, Marton TF, Logan DW, Flanagan K, Cruz JR, Saghatelian A, et al. Identification of protein pheromones that promote aggressive behaviour. Nature 2007; 450: 899902, doi: 10.1038/nature05997.

24. Agre P, Kozono D. Aquaporin water channels: molecular mechanisms for human diseases. FEBS Lett 2003; 555: 72-78, doi: 10.1016/S0014-5793(03)01083-4.

25. Zingg $\mathrm{HH}$, Laporte SA. The oxytocin receptor. Trends Endocrinol Metab 2003; 14: 222-227, doi: 10.1016/S10432760(03)00080-8.

26. Sozen I, Arici A. Interactions of cytokines, growth factors, and the extracellular matrix in the cellular biology of uterine leiomyomata. Fertil Steril 2002; 78: 1-12, doi: 10.1016/ S0015-0282(02)03154-0.

27. Malik M, Norian J, McCarthy-Keith $D$, Britten J, Catherino $\mathrm{WH}$. Why leiomyomas are called fibroids: the central role of extracellular matrix in symptomatic women. Semin Reprod Med 2010; 28: 169-179, doi: 10.1055/s-0030-1251475.

28. Islam MS, Protic O, Stortoni $P$, Grechi G, Lamana $P$, Petraglia F, et al. Complex networks of multiple factors in the pathogenesis of uterine leiomyoma. Fertil Steril 2013; 100: 178-193, doi: 10.1016/j.fertnstert.2013.03.007.

29. Zaitseva M, Vollenhoven BJ, Rogers PA. Retinoic acid pathway genes show significantly altered expression in uterine fibroids when compared with normal myometrium.
Mol Hum Reprod 2007; 13: 577-585, doi: 10.1093/molehr/ gam040.

30. Leppert PC, Baginski T, Prupas C, Catherino WH, Pletcher $\mathrm{S}$, Segars $\mathrm{JH}$. Comparative ultrastructure of collagen fibrils in uterine leiomyomas and normal myometrium. Fertil Steril 2004; 82: 1182-1187, doi: 10.1016/j.fertnstert.2004.04.030.

31. Levy G, Malik M, Britten J, Gilden M, Seagars J, Catherino $\mathrm{WH}$. Liarozole inhibits transforming growth factor-beta3mediated extracellular matrix formation in human threedimensional leiomyoma cultures. Fertil Steril 2014; 102: 272-281, doi: 10.1016/j.fertnstert.2014.03.042.

32. Lopez GN, Turck CW, Schaufele F, Stallcup MR, Kushner PJ. Growth factors signal to steroid receptors through mitogenactivated protein kinase regulation of $\mathrm{p} 160$ coactivator activity. J Biol Chem 2001; 276: 22177-22182, doi: 10.1074/jbc. M010718200.

33. Derynck R, Zhang YE. Smad-dependent and Smad-independent pathways in TGF-beta family signalling. Nature 2003; 425: 577-584, doi: 10.1038/nature02006.

34. Jeong YJ, Noh EM, Lee YR, Yu HN, Jang KY, Lee SJ, et al. $17 \beta$-estradiol induces up-regulation of PTEN and PPARgamma in leiomyoma cells, but not in normal cells. Int $J$ Oncol 2010; 36: 921-927, doi: 10.3892/ijo_00000571.

35. Nam DH, Ramachandran S, Song DK, Kwon KY, Jeon DS, Shin SJ, et al. Growth: inhibition and apoptosis induced in human leiomyoma cells by treatment with the PPAR gamma ligand ciglitizone. Mol Hum Reprod 2007; 13: 829-836, doi: $10.1093 / \mathrm{molehr} / \mathrm{gam} 071$. 